\title{
Propriétés physiques et mécaniques du bois de teck de provenances tanzanienne et locale au Bénin
}

\section{Montcho Crépin HounLONON ${ }^{1}$ \\ Clément A. KouchadE ${ }^{1}$ \\ Basile B. KOUNOUHEWA ${ }^{1}$}

${ }^{1}$ Université d'Abomey-Calavi (UAC)

Faculté des sciences et techniques (FAST)

Laboratoire de physique du rayonnement (LPR)

BP 526, Cotonou

Bénin

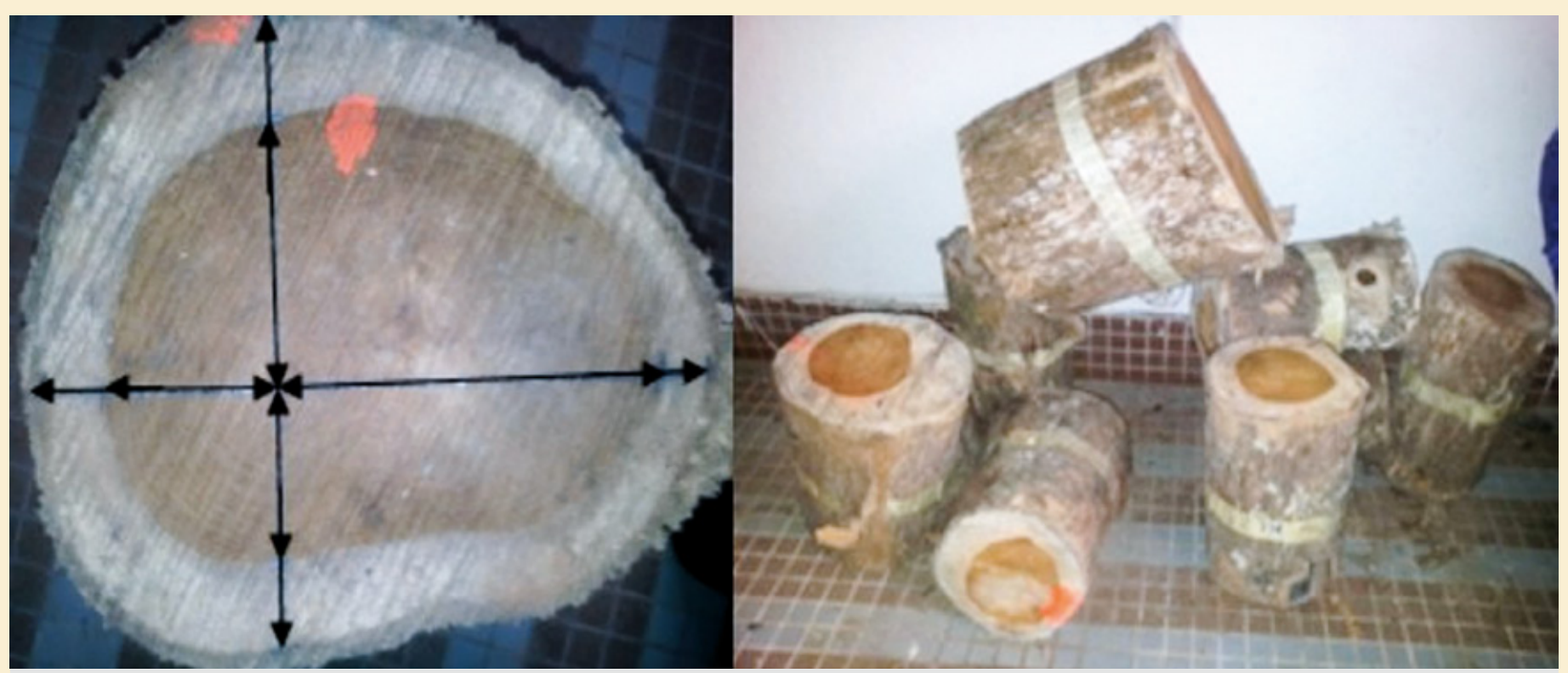

Photo 1.

Rondelles de teck montrant les rayons pour la détermination du pourcentage de bois de cœur. Photo M. C. Hounlonon. 


\section{C. Hounlonon, C. A. Kouchade, B. B. KOUNOUHEWA}

\section{RÉSUMÉ}

\section{PROPRIÉTÉS PHYSIQUES ET MÉCANIQUES DU BOIS DE TECK DE PROVENANCES TANZANIENNE ET LOCALE AU BÉNIN}

Le teck (Tectona grandis L. f.) est une espèce ligneuse tropicale dont le bois est très demandé. Au Bénin, la provenance tanzanienne et la provenance locale sont les deux variétés de cette espèce les plus plantées. Pour contribuer à la caractérisation du bois de teck du Bénin, nous avons déterminé et comparé, pour le bois de chaque provenance, les modules d'élasticité et de cisaillement, la longueur du fût, la circonférence et l'épaisseur d'écorce à hauteur d'abattage, le pourcentage de bois de cœur, la circonférence à une hauteur de $1,30 \mathrm{~m}$ du sol et la masse volumique (ou la densité) à $12 \%$ de teneur en eau. Les échantillons ont été prélevés dans les plantations de la Lama sur des arbres de 20 à 25 ans (six billes de provenance locale et quatre billes de provenance tanzanienne). Les résultats obtenus sur la base de cet échantillon indiquent que, pour cette classe d'âge, les valeurs de ces propriétés pour le bois de teck de provenance locale sont supérieures à celles de la provenance tanzanienne, sauf pour le pourcentage de bois de cœur. Sur la base de notre échantillonnage, le teck de provenance locale semble donc d'une certaine qualité, qu'il faut tendre à valoriser par la sélection biologique ou clonale des meilleurs sujets, aptes à s'adapter non seulement au changement climatique mais aussi aux structures des sols. Cela permettrait de favoriser la production du bois de teck sur toute l'étendue du territoire national, voire de la sous-région. Toutefois, la mise en place d'une variété hybride conciliant les atouts des deux provenances pourrait aussi être une grande avancée dans l'amélioration des essences exploitées au Bénin.

Mots-clés : teck, Tectona grandis, module d'élasticité, module de cisaillement, Lama, Bénin, Tanzanie.

\section{ABSTRACT}

\section{PHYSICAL AND MECHANICAL PROPERTIES OF TEAK WOOD FROM TANZANIAN AND LOCAL PROVENANCES IN BENIN}

Teak (Tectona grandis L. f.) is a tropical timber species which is in great demand. In Benin, the two most commonly planted teak varieties are of Tanzanian and local provenances. To contribute to the characterisation of teak from Benin, we determined and compared, for each provenance, the elasticity and shear modulus, height of the bole, the circumference and bark thickness at felling height, the percentage of heartwood, the circumference $1.30 \mathrm{~m}$ from the ground and the density of the wood at a $12 \%$ moisture content. The samples were taken in the Lama plantations from trees of 20 to 25 years of age (6 logs of local provenance 4 of Tanzanian provenance). The results obtained with these samples indicate that for this age class, the values for these properties are higher for the local teak than for the Tanzanian teak, except for the percentage of heartwood. Based on our sample, therefore, the local teak wood is of a fair quality that should be enhanced through biological selection or cloning of the specimens best able to adapt not only to climate change but also to soil structure. This would help to promote teak production across the entire country and even the sub-region. However, establishing a hybrid variety that combines the advantages of both provenances could also be a major step towards improving all tree species used in Benin.

Keywords: teak, Tectona grandis, elasticity modulus, shear modulus, Lama, Benin, Tanzania.

\section{RESUMEN}

\section{PROPIEDADES FISICOMÉCANICAS DE LA MADERA DE TECA DE ORIGEN TANZANO Y LOCAL EN BENÍN}

La teca (Tectona grandis L. f.) es una especie leñosa tropical cuya madera tiene una gran demanda. En Benín, la procedencia tanzana y la local son las dos variedades de esta especie más plantadas. Para contribuir a la caracterización de la madera de teca de Benín, se procedió a determinar y comparar, en la madera de ambas procedencias, los módulos de elasticidad y rigidez, la longitud de fuste, la circunferencia y grosor de la corteza a la altura de corta, el porcentaje de duramen, circunferencia a 1,30 m del suelo y peso específico (o densidad) al $12 \%$ de humedad. Las muestras se tomaron en las plantaciones de la Lama en árboles de 20 a 25 años (6 trozas de procedencia local y 4 de procedencia tanzana). Los resultados obtenidos basándose en esta muestra indican que, en este rango de edad, la madera de teca de procedencia local muestra unos valores superiores a los de la procedencia tanzana en todas las propiedades excepto en el porcentaje de duramen. Tomando como referencia nuestro muestreo, la teca de procedencia local parece que tiene una cierta calidad que debe desarrollarse mediante selección biológica o clonal de los mejores individuos para que sean capaces de adaptarse no sólo al cambio climático sino también a las estructuras del suelo. Esto impulsaría la producción de madera de teca en todo el territorio nacional o en la subregión. Sin embargo, la creación de una variedad híbrida que reúna las ventajas de ambas procedencias también podría suponer un gran adelanto en el mejoramiento de las especies explotadas en Benín.

Palabras clave: teca, Tectona grandis, módulo de elasticidad, módulo de rigidez, Lama, Benín, Tanzania. 


\section{Introduction}

Le teck est une espèce ligneuse tropicale dont le bois est très apprécié et utilisé au niveau mondial en raison de ses propriétés technologiques exceptionnelles. Au Bénin, les premières plantations datent de la période coloniale. Les toutes premières plantations en Afrique de l'Ouest ont commencé au Nigeria, en 1902, avec des semences provenant d'abord de l'Inde et de Myanmar (Pandey et Brown, 2000 ; Ali, 2002). Le teck aurait été introduit plus tard au Bénin en 1916, par des missionnaires catholiques à partir de graines venues du Nigeria (Demenois et Heurtaux, 2001). Ganglo et al. (1999) évoquent de premières plantations domaniales de grande envergure au Bénin en 1949. Jusqu'en 1990, date à laquelle la provenance de teck dite tanzanienne a été introduite au Bénin, les semences à l'origine de plus de 10000 ha de plantations de teck dans les domaines classés de l'État provenaient des anciennes teckeraies mises en place par les colons.

Les premières plantations de teck ont permis la vulgarisation de l'essence à la faveur de ses caractéristiques technologiques, de la facilité des traitements sylvicoles, de ses usages multiples offerts à partir des premières éclaircies, de sa régénération aisée, et surtout d'une faible durée de rotation d'environ 40 ans. La provenance tanzanienne a été introduite en 1990 par l'Office national du bois du Bénin (ONAB), structure étatique en charge de la gestion des plantations domaniales de l'État, après un séjour d'un agent en Tanzanie dans la forêt de Kihuhwi, province de Tanga ( $5^{\circ} 12^{\prime}$ de latitude Sud et $38^{\circ} 39^{\prime}$ 'de longitude Est), à 260 m d'altitude (Azankpan et al., 2009 ; Ali, 2002). Cette provenance connaît aujourd'hui une assez bonne réussite auprès des planteurs publics et privés béninois et de la sous-région, en raison de son aspect physique et morphologique laissant envisager des qualités supérieures à la variété locale. Ses atouts observés dans les plantations sont sa croissance rapide, la rectitude de son fût, sa hauteur plus grande et son diamètre plus gros que ceux du teck local, sa ramification tardive, mais aussi l'absence de floraison précoce, au contraire observée chez le teck local (Azankpan et al., 2009).

Bien que le Bénin représente la porte d'entrée du teck tanzanien en Afrique de l'Ouest, pratiquement aucune étude n'est disponible sur les qualités du bois de ces deux provenances. Les travaux de Kokutse et al. (2009) sur la problématique de la performance du teck de provenance tanzanienne par rapport au teck local en plantation au Togo et ceux de Azankpan et al. (2009) au Bénin constituent aujourd'hui de rares références. Plus généralement, après un siècle de culture, aucune étude significative n'est disponible sur la qualité du bois de teck du Bénin, a fortiori sur la qualité du bois de teck de l'ONAB.

La demande croissante, les politiques et les législations forestières en vigueur dans les pays producteurs (Inde, Thaïlande, Laos) tendent à faire disparaître du marché le teck issu des espaces naturels. Celui-ci est progressivement remplacé par le teck de plantations relevant de diverses provenances, exploité selon des rotations de plus en plus courtes. Cette situation engendre une variabilité des qualités technologiques du bois de teck. L'insuffisance de la connaissance des propriétés de ce bois issu de plantations entraîne des polémiques sur la qualité de ses produits dérivés alimentant le marché international (Krishnapillay, 2000). Qualifiées à tort ou à raison de « bois aux qualités technologiques médiocres " comparativement au teck de forêt naturelle, les plantations de teck représentent aujourd'hui l'une des meilleures opportunités de production tropicale de bois d'œuvre dans plusieurs pays africains, notamment le Bénin, la Côte d'Ivoire, le Nigeria, la Tanzanie et le Togo.

Afin de caractériser le teck produit au Bénin, l'ONAB et le Laboratoire de physique du rayonnement ont mené une étude comparative des propriétés physiques et mécaniques des deux provenances, locale et tanzanienne. Ont été principalement investiguées les propriétés dendrométriques telles que la masse volumique (ou la densité) à $12 \%$ de teneur en eau, les modules d'élasticité et de cisaillement, et le pourcentage de bois de cœur. Ces propriétés sont les plus utiles à la caractérisation physique et mécanique du bois. La densité du bois, notamment, est un excellent indicateur de sa résistance mécanique. Le module d'élasticité du bois stabilisé à une humidité théorique de $12 \%$ reste une propriété mécanique de référence caractérisant la proportionnalité entre la charge et la déformation. II constitue un indicateur de la rigidité du bois au même titre que le module de cisaillement. La formation du bois de cœur est un caractère économique très important chez le teck, ce bois étant très durable et présentant des qualités décoratives très attrayantes pour la fabrication de produits finis de qualité supérieure (Kokutse et al., 2009).

La longueur du fût, la circonférence à hauteur d'abattage, l'épaisseur d'écorce à hauteur d'abattage et la circonférence à une hauteur de $1,30 \mathrm{~m}$ du sol constituent des propriétés dendrométriques complémentaires pouvant aider à la décision. C'est pourquoi une étude comparative relevant de ces paramètres s'est avérée nécessaire pour mieux conseiller les producteurs du bois de teck dans le choix des provenances à planter.

Cette étude avait pour but principal d'améliorer les connaissances sur le teck local béninois et celui de provenance tanzanienne, afin de fournir des références pour orienter à terme l'amélioration génétique des arbres, ce par sélection des individus présentant les meilleures aptitudes technologiques. 


\section{Matériels et méthodes}

\section{Cadre d'étude}

L'étude porte sur du bois de teck prélevé dans les plantations domaniales de l'ONAB, au sein de parcelles situées dans les secteurs forestiers de Akpè (commune de Toffo, département de l'Atlantique), Koto et Agrimey (commune de Zogbodomè, département du Zou) ainsi que Djigbé (commune de Zê, département de l'Atlantique) (figure 1).

Les échantillons ont été prélevés dans la zone de transition entre le climat subéquatorial et le climat tropical. Cette zone est caractérisée par une grande saison pluvieuse de mars à juin et une petite de septembre à novembre, ainsi qu'une grande saison sèche de novembre à février et une petite saison sèche de juillet à août (ONAB, 2011). La pluviosité moyenne annuelle est de 1100 mm (ONAB, 2011). Elle présente des variations interannuelles, avec des années d'inondation ou de sécheresse, mais aussi d'un secteur à l'autre. La température moyenne annuelle varie entre $25^{\circ} \mathrm{C}$ et $29^{\circ} \mathrm{C}$ avec des maxima thermiques enregistrés en saison sèche (février et mars) atteignant $40^{\circ} \mathrm{C}$ et des minima de $22{ }^{\circ} \mathrm{C}$, enregistrés en période d'harmattan (décembre). L'humidité relative est constamment élevée. L'insolation est de sept heures par jour en moyenne en saison sèche, mais peut descendre à 3,5 heures en moyenne par jour aux mois d'août et de septembre (ONAB, 2011).
Le bassin sédimentaire du Sud-Bénin, qui recouvre la zone d'étude, est un ensemble de formations reposant en discordance sur le sol granito-gneissique. Certains secteurs, principalement Akpé et Koto, se présentent dans la dépression argilo-marneuse dite de la Lama où le sol est argileux, profond et acide, avec un pH compris entre 5 et 5,5 dans tout le profil, sauf parfois en surface où il varie entre 6,5 et 7 . Ces formations sont caractérisées par quatre types de vertisols : les vertisols argileux sur calcaire à faible profondeur, les vertisols argileux à calcaires, les vertisols argileux, et les sols argileux à recouvrement sableux (Hincourt et Drechsel, 1995).

\section{Méthodes}

Nous avons prélevé des billes de 1,5 m de hauteur pour la caractérisation mécanique, et des rondelles pour l'estimation du pourcentage de bois de cœur. Chaque échantillon a été marqué d'un code d'identification indiquant sa provenance : - à Koto, deux billes de la provenance locale (23 et 25 ans), et deux de la provenance tanzanienne (23 et 24 ans) ;

- à Akpè, deux billes de la provenance locale (20 ans) et deux de la provenance tanzanienne (20 et 21 ans) ; - à Agrimey, une bille de la provenance locale (23 ans) ;

- à Djigbé, une bille de la provenance locale (23 ans).

\section{Mesure des caractéristiques dendrométriques et du pourcentage de bois de cœur}

Les caractéristiques dendrométriques mesurées étaient la longueur du fût, la circonférence à hauteur d'abattage, l'épaisseur d'écorce à hauteur d'abattage, et la circonférence à une hauteur de $1,30 \mathrm{~m}$ du sol.

Pour mesurer le pourcentage de bois de cœur, trois rondelles d'environ $5 \mathrm{~cm}$ d'épaisseur ont été prélevées sur chaque arbre abattu : une à la base du tronc, une à $1,30 \mathrm{~m}$ de hauteur du tronc, et la dernière à la base du houppier. Les rayons de la moelle à l'écorce et de la moelle à la limite du bois de cœur ont été mesurés sur chaque rondelle (photo 1). Les surfaces totales de la section (surface totale de la rondelle notée) et du bois de cœur (surface du duramen notée) ont été calculées pour chaque rondelle en utilisant la moyenne quadratique des rayons (Berthier et al., 2001 ; Kokutse, 2002). Selon Berthier et ses collaborateurs, quatre rayons orthogonaux permettent d'avoir les mêmes résultats. 
Ainsi, la surface totale se calcule comme suit :

$S_{t}=\frac{\pi}{4}\left(\sum_{i=1}^{4} R_{i}^{2}\right)(1)$

La surface du duramen ou du bois de cœur est :

$S_{H W}=\frac{\pi}{4}\left(\sum_{i=1}^{4} R_{i}^{2}\right)(2)$

Le pourcentage $P$ de bois de cœur a été calculé par rapport à la surface totale de la rondelle :

$P=100 \frac{S_{H W}}{S_{t}}$

Des analyses de variance ont été réalisées pour déterminer la variation du taux de bois de cœur formé, en fonction de la provenance du bois.

\section{Mesure des caractéristiques physiques et mécaniques}

\section{Mesure de la densité à $12 \%$ d'humidité}

La masse volumique (ou la densité) à $12 \%$ de teneur en eau est une caractéristique technologique de base, la première à déterminer pour qualifier un bois. Dans les billes prélevées, des planchettes ont été débitées en éprouvettes de $500 \mathrm{~mm} \times 25 \mathrm{~mm} \times 25 \mathrm{~mm}$, en respectant, dans la mesure du possible, les directions longitudinale, tangentielle et radiale. Chaque bille a donné une cinquantaine d'éprouvettes. La position des éprouvettes par rapport à la moelle ou par rapport à l'aubier n'a pas été déterminée. La densité du bois a été déterminée à l'aide d'une balance de pesée (précise à 0,01 g) pour la mesure de la masse, et d'un pied à coulisse pour la mesure des cotes de l'éprouvette, la longueur étant normalisée à $500 \mathrm{~mm}$. La formule (4) a été utilisée pour calculer la densité de chaque éprouvette :

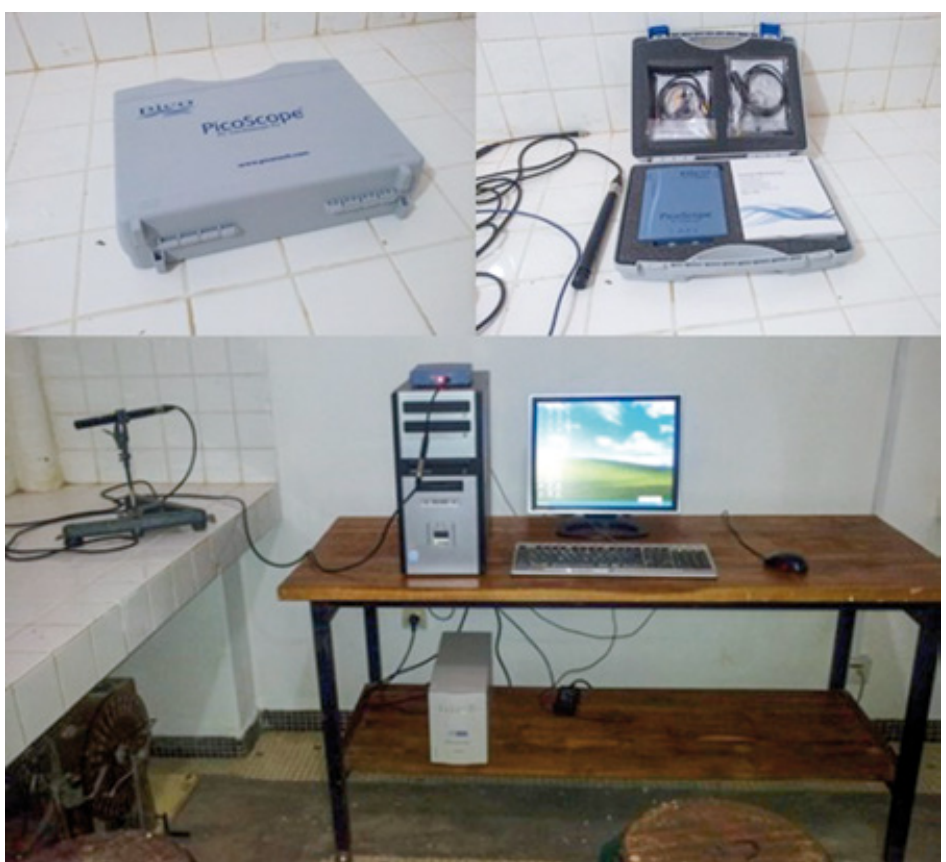

Photo 2.

Dispositif de caractérisation mécanique du bois. Photo M. C. Hounlonon.
$D=\frac{M}{V} \operatorname{avec} V=$ L.l.e

Où $D$ est la masse volumique, $M(\mathrm{~kg})$ est la masse de l'échantillon, $V$ est le volume de l'échantillon après stabilisation à $12 \%$, et $L(\mathrm{~m}), \mathrm{l}(\mathrm{m})$ et e $(\mathrm{m})$ sont respectivement la longueur, la largeur moyenne et l'épaisseur moyenne de l'éprouvette.

Mesure du module d'élasticité et du module de cisaillement

Le module d'élasticité et le module de cisaillement ont été mesurés à partir des mêmes éprouvettes que celles ayant servi à mesurer la densité du bois. Le dispositif de la photo 2 a été utilisé pour le test. Le dispositif était constitué d'un microphone (LEM, type EMU 4535), d'une carte d'acquisition (Pico Technology Type PicoScope 3224), d'un ordinateur, et d'un système élastique sur lequel était disposé le matériau bois à caractériser. Le système était piloté par le logiciel BING (Beam Identification by Non-destructive Grading), initialement développé pour évaluer le matériau bois, actuellement dans sa version 9.

La méthode a été mise au point au Cirad (Baillères et al., 1998). Le principe de la mesure repose sur l'analyse spectrale des vibrations naturelles de flexion. Ce procédé permet de déterminer les fréquences propres d'une poutre à partir de sa réponse à une excitation appliquée à l'une des extrémités, de façon à solliciter simultanément tous les modes propres de vibration. La réponse est enregistrée à l'autre extrémité au moyen d'un microphone. Les vibrations sont ensuite transmises à un ordinateur. Le signal est traité par une procédure FFT (transformée de Fourier rapide). Un ajustement sur les premiers modes, basé sur la théorie de Timoshenko sur les poutres flottantes, permet de déterminer les modules d'élasticité et de cisaillement (Baillères et al., 1998).

Pour le test, les échantillons sont posés sur les deux supports en élastique afin qu'une simple percussion à une extrémité de l'échantillon provoque une vibration assez libre. Les appuis qui constituent les nœuds de vibration sont respectivement positionnés au quart et aux trois quarts de la longueur de l'échantillon. Sur ces supports, l'échantillon est placé de manière à ce que la direction tangentielle soit verticale et représente sa hauteur dans les calculs. La percussion sur une extrémité de l'échantillon se réalise par l'opérateur, grâce à une barre portant une boule à l'une de ses extrémités (Brancheriau et al., 2010).

Dans le cadre de cette étude, le test était en flexion et la percussion a été faite sur la face supérieure de l'échantillon. Le microphone était placé verticalement à l'autre extrémité de l'échantillon et permettait d'enregistrer l'onde sonore émise. Cette dernière était transmise à l'ordinateur via la carte d'acquisition reliée à un filtre passe-bas. Le logiciel BING a permis de traiter numériquement le signal et d'utiliser les données géométriques de l'échantillon ainsi que sa masse pour déterminer ses caractéristiques mécaniques. De toutes ces caractéristiques mesurées, seuls les modules de Young et de cisaillement déterminés suivant la méthode de Timoshenko nous intéressaient dans cette étude. Cet essai avait la particularité d'être non destructif. 


\section{Tableau I.}

Comparaison des propriétés dendrométriques et du pourcentage de bois de cœur de tecks de provenance locale du Bénin et tanzanienne, âgés de 20 à 25 ans. Les moyennes par colonne entre essences de provenances différentes suivies de la même lettre ne sont pas significativement différentes au seuil de $5 \%$.

\section{- nd \\ Provenance tanzanienne \\ Provenance locale du Bénin $\mathrm{p}$ \\ $0,21 \mathrm{~ns}$

\begin{tabular}{|c|c|}
\hline \multicolumn{1}{|c|}{$\operatorname{LF}(\mathbf{m})$} \\
\hline $\mathbf{m}$ & $\mathbf{s e}$ \\
\hline $10,45 \mathrm{a}$ & 0,40 \\
\hline $11,04 \mathrm{a}$ & 0,26 \\
\hline
\end{tabular} \\ Résultats \\ Caractéristiques dendrométriques et pourcentage de bois de cœur}

$\mathrm{m}$ : moyenne ; se : erreur standard ; *** : différence significative au seuil de $0,1 \%$; ns : non significative ; LF : longueur du fût après abattage ; Cir-ab : circonférence à hauteur d'abattage ; Cir $(1,30)$ : circonférence à hauteur d'homme ; Ep-ec : épaisseur écorce ; BC : pourcentage bois de cœur ; BCH : pourcentage bois de cœur sous houppier.
Les résultats du tableau I montrent qu'il n'y a pas de différence significative dans les caractéristiques dendrométriques des tecks de provenance locale et tanzanienne ayant 20 à 25 ans d'âge. Cependant, en moyenne, la longueur du fût après abattage et les circonférences à hauteur d'homme de la provenance locale sont supérieures à celles de la provenance tanzanienne à cet âge (figure 2). Ce n'est pas le cas du pourcentage du bois de cœur dont la valeur pour le bois tanzanien est supérieure à celle du bois local.

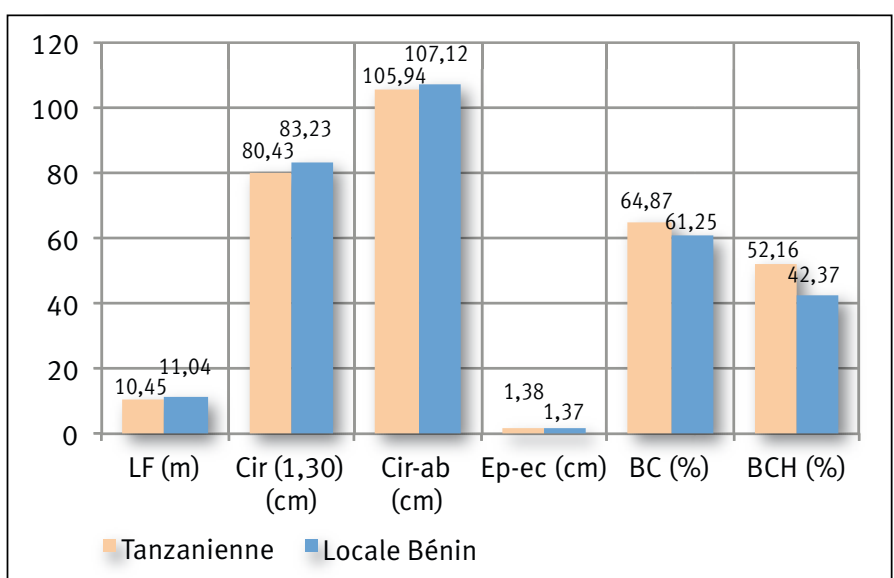

Figure 2.

Comparaison des propriétés dendrométriques et du pourcentage de bois de cœur des tecks de provenance locale du Bénin et tanzanienne, âgés de 20 à 25 ans.

$\mathrm{LF}$ : longueur du fût après abattage ; Cir-ab : circonférence à hauteur d'abattage ; $\operatorname{Cir}(1,30)$ : circonférence à hauteur d'homme; Ep-ec : épaisseur écorce ; BC : pourcentage bois de cœur ; $\mathrm{BCH}$ : pourcentage bois de cœur sous houppier.

\section{Propriétés physiques et mécaniques des tecks des provenances locale et tanzanienne}

La figure 3 (tableau II) montre que la densité des bois de teck de provenance locale et tanzanienne âgés de 20 à 25 ans est semblable. Il n'y a donc pas de différence significative entre la densité de ces deux types de bois $(P>0,05)$. De par la valeur de ces densités, les bois de provenance locale et tanzanienne sont des bois mi-lourds, donc mi-durs. Cependant, le module d'élasticité et le module de cisaillement de ces deux types de bois sont significativement différents au seuil de $1 \%$. Les bois de provenance locale présentent un module d'élasticité et un module de cisaillement supérieurs à ceux de provenance tanzanienne. Bien qu'appartenant tous à la classe de module moyen, les bois de provenance locale sont plus rigides que ceux d'origine tanzanienne.

\section{Discussion}

L'ensemble des résultats obtenus dans cette étude, basée certes sur un faible nombre d'arbres investigués ne nous permettant pas d'établir des conclusions définitives ni générales, montrent que le bois de teck de provenance locale présente des caractéristiques physiques et mécaniques supérieures à celles du bois de provenance tanzanienne, dont les qualités ont été pourtant vantées et continuent d'être mises en avant au Bénin et dans la sous-région, notamment au Togo et au Nigeria.

En dehors du pourcentage de bois de cœur qui discrimine positivement le bois de teck de provenance tanzanienne, la provenance locale la supplante en ce qui concerne la longueur du fût, la circonférence à hauteur d'abattage, l'épaisseur d'écorce à hauteur d'abattage, la circonférence à une hauteur de $1,30 \mathrm{~m}$ du sol, la masse volumique (ou la densité) à $12 \%$ de teneur en eau, le module d'élasticité et le module de cisaillement. Cette dernière tendance avait été observée par Kokutse et al. (2009) sur des arbres de sept ans. Les maxima de pourcentage de bois de cœur obtenus dans leurs travaux donnaient des valeurs maximales de $31,5 \%$ pour la provenance tanzanienne et de $21,7 \%$ pour la provenance locale à 1,30 m du sol, s'agissant d'arbres de sept ans. 
Tableau II.

Comparaison de la masse volumique, du module d'élasticité et du module de cisaillement de tecks de provenance locale du Bénin et tanzanienne, âgés de 20 à 25 ans. Les moyennes par colonne entre essences de provenance différente suivies de la même lettre ne sont pas significativement différentes au seuil de $5 \%$.

\begin{tabular}{|c|c|c|c|c|c|c|}
\hline \multirow[b]{3}{*}{ Provenance locale du Bénin } & \multicolumn{2}{|c|}{ Masse volumique $\left(\mathrm{kg} / \mathrm{m}^{3}\right)$} & \multicolumn{2}{|c|}{ Module d’élasticité (MPa) } & \multicolumn{2}{|c|}{ Module de cisaillement ( $\mathrm{MPa})$} \\
\hline & m & se & m & se & m & se \\
\hline & $799,90 a$ & 9 & $14628 a$ & 387,10 & $1481 a$ & 79,62 \\
\hline Provenance tanzanienne & $799,73 a$ & 7,51 & $12913 b$ & 239,44 & $1022,18 b$ & 27,79 \\
\hline P-value & $0,98 \mathrm{~ns}$ & & $0,000^{\star \star \star}$ & & $0,000^{\star \star \star}$ & \\
\hline
\end{tabular}

Nos travaux ont non seulement confirmé cette aptitude de la provenance tanzanienne, mais aussi attesté que cette tendance s'affirme avec l'âge. Ainsi, pour les échantillons entrés dans notre étude et dont l'âge varie de 20 à 25 ans, le pourcentage de bois de cœur de la provenance tanzanienne se révèle supérieur à celui de la locale, de $6 \%$ à 1,30 m du sol, et de $19 \%$ sous le houppier. Ceci fournit des informations supplémentaires sur les possibilités d'exploitation de la provenance tanzanienne. Avec ce fort pourcentage de bois de cœur sous le houppier, pratiquement la totalité du tronc de l'arbre de provenance tanzanienne est utile à l'exploitation. Le pourcentage de bois de cœur étant un critère de choix et d'estimation de la qualité du bois de teck, il paraît pertinent de conférer au teck tanzanien une qualité meilleure que celle de la provenance locale.

Cependant, d'autres caractéristiques dendrométriques restent en faveur des provenances locales. La longueur du fût après abattage et les circonférences à hauteur d'homme de la provenance locale sont en effet supérieures à celles

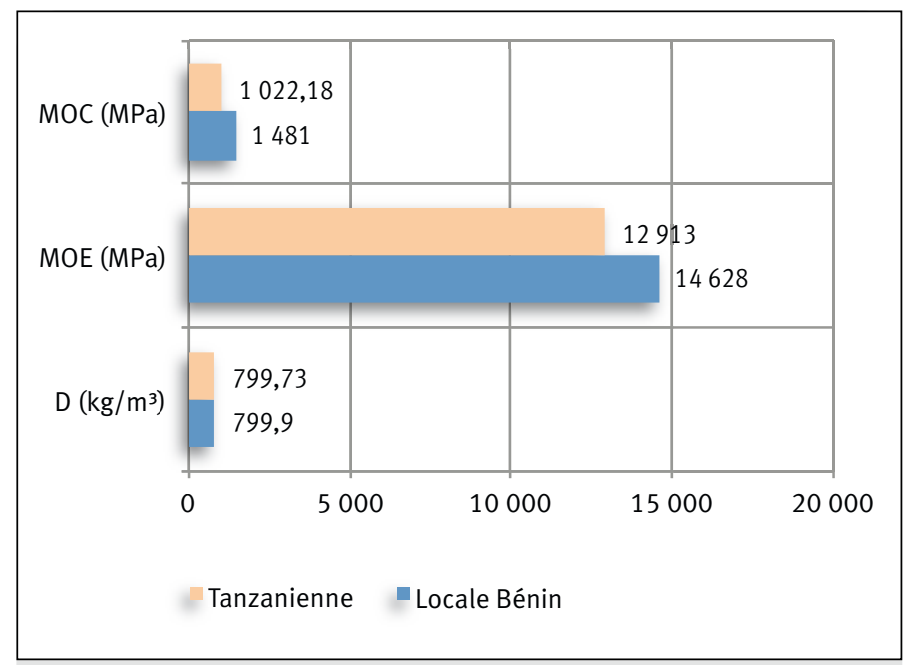

Figure 3.

Comparaison de la masse volumique, du module d'élasticité et du module de cisaillement des tecks de provenance locale du Bénin et tanzanienne, âgés de 20 à 25 ans.

$\mathrm{D}$ : masse volumique ; $\mathrm{MOE}$ : module d'élasticité ; $M O C$ : module de cisaillement. de la provenance tanzanienne de 20 à 25 ans, comme cela avait été observé par Kokutse et al. (2009) sur des arbres de sept ans au Togo. Par contre, les résultats obtenus par ces mêmes auteurs sur la caractérisation mécanique du bois de teck des deux provenances sont en contradiction avec nos résultats. En effet, le module d'élasticité du teck de provenance locale âgé de 20 à 25 ans (de l'ordre de $14500 \mathrm{MPa}$ en moyenne) rend compte d'un bois plus rigide que celui de la provenance tanzanienne du même âge (environ $13000 \mathrm{MPa}$ en moyenne) ; or, Kokutse et al. (2009) ont observé que la provenance tanzanienne était légèrement plus rigide que la provenance locale à sept ans. Les résultats obtenus par ces auteurs restent cependant très variables, du fait d'un échantillonnage plus étendu que le nôtre, et établi de manière aléatoire. Pour ce qui nous concerne, les arbres échantillonnés étaient peu nombreux et les arbres choisis dans les deux provenances étaient manifestement d'une certaine qualité, s'agissant d'arbres à fût droit. Les travaux de Azankpan et al. (2009) sur les arbres de mêmes provenances et de la même forêt de la Lama, mais âgés de 16 à 22 ans, ont également révélé une nette supériorité du teck tanzanien sur le teck local, surtout pour la vigueur, la productivité et la qualité du fût.

Le tableau III présente les corrélations significatives au seuil de $0,1 \%$, de $1 \%$ et de $5 \%$ entre les différents paramètres étudiés sur tous les échantillons préparés. Au seuil de $5 \%$, la corrélation est significative entre la densité et le module d'élasticité ; au seuil de $1 \%$, la corrélation est significative entre la densité et la circonférence à hauteur d'abattage, la corrélation entre la densité et l'une ou l'autre des autres caractéristiques n'étant cependant pas significative. Toutefois, aucune corrélation significative n'existe entre le module d'élasticité des bois de teck étudiés et les autres propriétés retenues, sauf la longueur de fût et les circonférences. La provenance n'a pas d'effet sur la rigidité du bois de teck. Ce résultat a été confirmé par Sekar (1972), qui avait relevé l'absence de corrélation significative entre la vitesse de croissance et la solidité du bois. D'autres études ont indiqué que la densité et les propriétés mécaniques du bois étaient sans rapport avec la vitesse de croissance (par conséquent avec la provenance), ou que les arbres à croissance rapide d'espèces à pores diffus avaient un bois plus dense et plus solide (Harris, 1981; Bhat et al., 1987; Rajput et al., 1991). 


\begin{tabular}{|c|c|c|c|c|c|c|c|c|}
\hline \multicolumn{9}{|c|}{$\begin{array}{l}\text { Tableau III. } \\
\text { Corrélation entre les paramètres physiques, mécaniques et les paramètres dendrométriques de tecks } \\
\text { âgés de } 20 \text { à } 25 \text { ans. }\end{array}$} \\
\hline MOE & $0,21^{*}$ & & & & & & & \\
\hline MOC & $0,17 \mathrm{~ns}$ & $0,01 \mathrm{~ns}$ & & & & & & \\
\hline LF & $-0,15 n s$ & $0,43^{\star * \star}$ & $-0,07$ ns & & & & & \\
\hline $\operatorname{Cir}(1,30)$ & $-0,04 \mathrm{~ns}$ & $0,24^{\star \star}$ & $-0,06 \mathrm{~ns}$ & $0,24^{\star \star}$ & & & & \\
\hline Cir-ab & $-0,24^{\star \star}$ & $0,31^{\star \star \star}$ & $-0,04 \mathrm{~ns}$ & $0,15 \mathrm{~ns}$ & $0,77^{\star \star \star}$ & & & \\
\hline Ep-ec & $0,02 \mathrm{~ns}$ & $-0,16 \mathrm{~ns}$ & $0,16 \mathrm{~ns}$ & $0,42^{\star \star \star}$ & $-0,13 \mathrm{~ns}$ & $-0,16 \mathrm{~ns}$ & & \\
\hline $\mathrm{BC}$ & $-0,17 \mathrm{~ns}$ & $0,001 \mathrm{~ns}$ & $-0,33^{\star \star \star}$ & $-0,03 \mathrm{~ns}$ & $0,58^{\star \star \star}$ & $0,59 * \star \star$ & $-0,33^{\star \star \star}$ & \\
\hline $\mathrm{BCH}$ & $-0,17 \mathrm{~ns}$ & $0,01 \mathrm{~ns}$ & $-0,28^{\star \star}$ & $-0,38^{\star \star \star}$ & $0,14 \mathrm{~ns}$ & $0,36^{\star \star \star}$ & $-0,75^{\star \star \star}$ & $0,51^{\star \star \star}$ \\
\hline & $\begin{array}{l}\text { élation signifi } \\
\text { ns : non signi } \\
\text { irconférence } \\
\text { centage bois }\end{array}$ & $\begin{array}{l}\text { tive au seuil } \\
\text { ative; MOE } \\
\text { lauteur d'aba } \\
\text { cœur; } B C H\end{array}$ & $\begin{array}{l}0,1 \% ; * \star \\
\text { nodule d'élas } \\
\text { age ; Cir }(1,30 \\
\text { ourcentage b }\end{array}$ & $\begin{array}{l}\text { rrélation signi } \\
\text { ité ; MOC: mc } \\
\text { circonférence } \\
\text { s de cœur sou }\end{array}$ & $\begin{array}{l}\text { cative au seuil } \\
\text { lule de cisaille } \\
\text { hauteur d'ho } \\
\text { houppier. }\end{array}$ & $\begin{array}{l}\text { e } 1 \% \text {; * : cor } \\
\text { ent ; LF : long } \\
\text { me; Ep-ec : }\end{array}$ & $\begin{array}{l}\text { Elation signific } \\
\text { eur du fût aprè } \\
\text { aisseur écorce }\end{array}$ & $\begin{array}{l}\text { ive au seuil } \\
\text { abattage ; }\end{array}$ \\
\hline
\end{tabular}

Plus récemment, une étude sur les propriétés du bois de teck à croissance rapide d'âges différents poussant dans des plantations a montré que la densité en bois, le module de rupture, le module d'élasticité ou d'écrasement maximal étaient sensiblement les mêmes (Bhat, 1998). L'étude concluait que les jeunes arbres (de 13 à 21 ans) n'étaient pas nécessairement inférieurs aux arbres plus vieux (de 55 à 65 ans), du point de vue de la densité et de la solidité du bois ; par voie de conséquence, l'âge de rotation des tecks à croissance rapide pouvait être abaissé sans risque.

Il est compréhensible que les principaux critères d'adoption de la variété tanzanienne soient une croissance rapide et un tronc présentant peu de fourches, peu de cannelures et peu de bosses. Mais ces critères sont visuels et ne renseignent pas sur les propriétés intrinsèques $d u$ bois qui n'apparaîtront qu'après l'exploitation et la transformation. Le principal enseignement que l'on peut tirer de ce travail est que, contrairement à l'opinion générale, les principales caractéristiques technologiques du bois de teck de provenance tanzanienne, telles que la longueur du fût, la circonférence à hauteur d'abattage, l'épaisseur d'écorce à hauteur d'abattage, la circonférence à une hauteur de $1,30 \mathrm{~m}$ du sol, la masse volumique (ou la densité) à $12 \%$ de teneur en eau, le module d'élasticité et le module de cisaillement, ne sont pas forcément supérieures à celles du bois de teck de provenance locale.

Il reste à entreprendre une sélection biologique qui permettrait d'avoir, à partir des provenances locales, des sujets de grande performance morphologique, physique, mécanique et technologique. Il s'agirait d'assurer la promotion du teck local, adapté aux conditions écologiques et édaphiques de la sous-région, en recourant aux nouvelles technologies de sélection et de clonage, plutôt que de continuer à fonder l'espoir de nos plantations de teck sur une provenance qui ne présente pas de grands avantages s'agissant de la production d'un bois de qualité.
Les défis majeurs des plantations de tecks du Bénin sont non seulement liés à la morphologie des arbres mais aussi à leur résistance aux effets du changement climatique. La sélection doit donc allier caractéristiques morphologiques et résilience au changement climatique. Le croisement entre le teck local et le teck tanzanien peut représenter une solution, et la diffusion d'autres provenances sélectionnées lors des recherches pourrait aussi permettre de garantir la production d'un teck de meilleure qualité au Bénin.

\section{Conclusion}

Sur la base de notre échantillon de dix billes, il apparaît qu'entre 20 et 25 ans la longueur du fût, la circonférence à hauteur d'abattage, l'épaisseur d'écorce à hauteur d'abattage, la circonférence à une hauteur de $1,30 \mathrm{~m}$ du sol, la masse volumique (ou la densité) à $12 \%$ de teneur en eau, le module d'élasticité et le module de cisaillement du bois de teck de provenance locale présentent respectivement des valeurs supérieures à celles de la provenance tanzanienne, sauf pour le pourcentage de bois de cœur. Cette dernière propriété, liée à la valeur économique du bois, peut à elle seule induire la préférence des planteurs. Si nous y ajoutons les caractéristiques morphologiques externes du teck tanzanien telles que sa vigueur, sa forte productivité et la raideur de son fût, le producteur ne va pas hésiter à adopter cette variété. Mais il est fondamental de tenir compte des autres propriétés intrinsèques qui restent aussi des outils d'aide à la décision. C'est pourquoi il importe de valoriser davantage la variété locale à travers la sélection biologique ou clonale de meilleurs sujets pouvant répondre facilement aux exigences climatiques et édaphiques de notre milieu, surtout en contexte de changement climatique. II n'est pas exclu que la cohabitation des deux provenances ait déjà donné une variété hybride, aux caractéristiques meilleures. 
Les études de caractérisation devront se poursuivre afin de mieux évaluer l'effet de l'âge sur ces différentes propriétés. La question de l'âge d'exploitabilité demeure en effet aujourd'hui une réelle problématique qu'il s'agit d'intégrer.

\section{Remerciements}

Nous remercions l'Office national du bois représenté par son directeur général et ses collaborateurs pour le Fonds d'aide à la recherche forestière. Nous remercions également Loïc Brancheriau et Sébastien Paradis (Cirad).

\section{Références bibliographiques}

Ali S., 2002. Étude comparative de la croissance de deux provenances de teck (Tectona grandis L.f.) dans le système agrosylvicole Taugya à Haho-Baloé (Togo). Mémoire de l'École d'ingénieurs en agronomie, Université de Lomé, Togo, $68 \mathrm{p}$.

Azankpan J. D., Ganglo J. C., Zohoun S., 2009. Performances de deux provenances de teck (Tectona grandis L. f.) au Bénin. Annales des Sciences Agronomiques, 12 (1) : 55-65.

Baillères H., Calchera G., Demay L., Vernay M., 1998. Classement mécanique des bois guyanais de structure selon trois techniques non destructives. Bois et Forêts des Tropiques, 257 : 47-62. http://bft.cirad.fr/cd/ BFT 257 47-62.pdf

Berthier S., Kokutse A. D., Stokes A., Fourcaud T., 2001. Irregular heartwood formation in Maritime Pine (Pinus pinaster Ait): Consequences for biomechanical and hydraulic tree functioning. Annals of Botany, 87: 19-25.

Bhat K. M., 1998. Properties of fast-grown teakwood: impact on end-users' requirements. Journal of Tropical Forest Products, 4 (1): 1-10.

Bhat K. M., Bhat K. V., Dhamodaran T. K., 1987. A note on specific gravity difference between dominant and suppressed trees in teak. Indian Journal of Forestry, 10 (1): 61-62.

Brancheriau L., Kouchade C., Brémaud I., 2010. Internal friction measurement of tropical species by various acoustic methods. Journal of Wood Science, 56: 371-379.

Demenois J., Heurtaux A., 2001. La filière teck en Inde du Sud (Karnataka, Kérala, Tamil Nadu). De la plantation à la commercialisation du teck : aspects sociaux, économiques et techniques. Mémoire Engref/Cirad-Forêt/Institut français de Pondichéry, $117 \mathrm{p}$.

Ganglo J. C., Lejoly J., Pipar T., 1999. Le teck au Bénin, gestion et perspectives. Bois et Forêts des Tropiques, 261 (3) : 17-27. http://bft.cirad.fr/cd/BFT 261 17-27.pdf
Harris J. M., 1981. Effect of rapid growth on wood processing. In: Proceedings of the XVII IUFRO World Congress, Kyoto, Japan, 6-12 September 1981, 117-125.

Hincourt D., Drechsel P., 1995. Instruction pour la cartographie forestière des sites de la forêt classée de la Lama. DFS-Assistance technique du Projet « Développement de l'économie forestière et du bois ».

Kokutse A. D., Adjonou K., Kokou K., Gbeassor M., 2009. Problématique de la performance du teck de provenance tanzanienne par rapport au teck local en plantation au Togo. Bois et Forêts des Tropiques, 302 (4) : 43-52. http://bft. cirad.fr/cd/BFT 302 43-52.pdf

Kokutse A. D., 2002. Analyse de la qualité du bois de teck (Tectona grandis L.f.) en plantation au Togo : formation du bois de cœur, propriétés mécaniques et durabilité. Thèse de doctorat, Université Bordeaux I, France, 142 p.

Krishnapillay B., 2000. La sylviculture et la gestion des plantations de teck. Unasylva, 201 : 14-20.

ONAB, 2011. Plan directeur d'aménagement et de gestion du noyau central de la forêt classée de la Lama 2011-2020. Version de travail avec l'assistance du CENAGREF, 96 p.

Pandey D., Brown C., 2000. Le teck dans le monde. Unasylva, 201 : 3-13.

Rajput S. S., Shukla N. K., Lai M., 1991. Some studies on the variation of strength properties of Tectona grandis from Mizoram. Journal of Timber Development Association of India, 37 (2): 33-38.

Sekar A. C., 1972. A comparative study of strength properties of timber from natural and man-made forests. In: Proceedings of the Symposium on Man-Made Forest in India, Dehra Dun, Inde, 5: 29-32. 


\section{Instructions for authors}

Use these instructions ifyou are preparing a manuscript tosubmit to Bois et Forêts des Tropiques. Potential contributors are advised that careful attention to the details below will greatly assist the Editor and thus speed the processing of their manuscripts. Poorly prepared manuscripts will be returned to authors.

Bois et Forêts des Tropiques publishes papers in the important and wide field of the Tropical and Mediterranean Forests. Papers devoted to the results of original research as well as those which form significant reviews will be considered. Research papers normally should not exceed 6000 words of main text and should have no more than 40 references. Reviews should not exceed 8000 words of main text and should have no more than 80 references A cover letter is needed, showing what was investigated, where and how it was done, why the results are important, what is their innovative nature and transferability, and why the author has chosen Bois et Forêts des Tropiques for his manuscript. Note that it is possible to suggest names for review but also provide names which are not preferred.

Bois et Forêts des Tropiques considers all manuscripts on the strict condition that the manuscript is your own original work, and does not duplicate any other previously published work, in whole or in part, including your own previously published work; the manuscript has been submitted only to Bois et Forêts des Tropiques and is not under consideration or peer review or accepted for publication or in press or published elsewhere; the manuscript contains nothing that is abusive, defamatory, libellous, obscene, fraudulent, or illegal. For all manuscripts non-discriminatory language is mandatory. Sexist or racist terms must not be used.

The Editor will acknowledge receipt of the manuscript, provide it with a manuscript reference number and assign it to reviewers. The reference number of the manuscript should be quoted in all correspondence with Bois et Forêts des Tropiques.

Papers are first inspected for suitability by the Editor or an editorial board member. Those suitable papers are then critically reviewed by usually two or three expert persons. On their advice the Editor provisionally accepts, or rejects, the paper. If acceptance is indicated the manuscript is usually returned to the author for revision. In some cases a resubmission is invited and on receipt of the new version, the paper may be sent to a third referee. If the author does not return the revised or resubmitted version within 60 days the paper will be classified as rejected. Final acceptance is made when the manuscript has been satisfactorily revised.

\section{General guidelines}

Manuscripts are accepted in French or English. For English manuscripts, British English spelling and punctuation are preferred. Manuscripts should be prepared according to the following structure:

Page 1. Title page. This should contain (a) the full title, preferably of less than 15 words and usually containing the geographical location of the study; (b) a running title of not more than 48 letters and spaces; (c) a list of up to 8 key words, separated by commas, in alphabetical order suitable for international retrieval systems;

Page 2. Authors page. The page should contain full names, affiliations (department, university), postal addresses, telephone numbers and email addresses of each author. Please provide an institutional email address for each author. One author should be identified as the corresponding author.

Page 3. Abstract. This should be a single paragraph, in passive mode, no more than 275 words long, concise summary of the paper intelligible on its own in conjunction with the title, without abbreviations or references. The abstract is written in the same language used in the text.

Page 4. et sea. Text. The main body of the text may contain the following sections in the sequence indicated: (a) Introduction, (b) Methods, (c) Results, (d) Discussion, (e) Conclusion, (f) Acknowledgements, (g) Literature Cited, (h) Appendices (as appropriate), (i) Tables with caption(s), (j) figure caption(s) as a list, (k) photograph captions as a list.

Tables (preferably in MS Word, they must not be submitted as images) should be provided either at the end of the manuscript or as separate files. Tables should be numbered consecutively with Latin numerals (for example: table I) and every table should be cited at least once in the text, in consecutive order.

Figures should be submitted as separate files in TIF or EPS format but captions to figures should be supplied on a separate sheet at the end of the main manuscript. All figures must be cited in consecutive order and are numbered consecutively with Arabic numerals (for example: figure 1). The actual figures should be supplied in a separate file. Ensure you have permission to use any figures or photos you are reproducing from another source. In addition, provide in separate Excel files the source of the graphics, to enhance figures during the publication process.

Photos are strongly recommended. Their number should range from 3 to 6 . They must be numbered, with Arabic numerals (for example: photo 1), in the order in which they appear in the text. Photograph files are in $10 \mathrm{~cm} \times 15 \mathrm{~cm}$ format, with a minimal resolution of $300 \mathrm{dpi}$, and must be compressed into JPEG at maximum quality.

Tables, figures and photos: Indicate in the text where the tables and figures should appear, for example by inserting [Table I near here]. All the captions have to be informative and explicit. Acknowledgements should be brief. Please supply all details required by any funding and grant-awarding bodies.

Notes should be avoided if at all possible; any notes will be printed as footnotes and not at the end of the paper.

Scientific names - The complete Latin name (genus, species and authority) must be given in full for every organism when first mentioned in the text unless a standard nomenclatural reference is available which can be cited. Authorities might alternatively appear in Tables where they are first used. Names of taxa at generic rank and below should be in italics.

\section{Advice to authors on preparing a manuscript}

Font: Times New Roman, 12 point. Double spacing must also be used throughout, allowing wide margins (about $3 \mathrm{~cm}$ ) on all sides. Main text pages are numbered. All pages of text have line numbers.

Section headings should be concise.

The corresponding author must be authorized by all co-authors to act as an agent on their behalf in all matters pertaining to publication of the manuscript, and the order of names should be agreed by all authors.

Anonymity for peer review: Ensure your identity and that ofyour co-authors is not revealed in the text of your article or in your manuscript files when submitting the manuscript for review. Headings: Please indicate the level of the section headings in your article.

If your article is accepted for publication, it will be copy-edited and typeset in the correct style for the journal.

\section{References style}

In the text

One author: Smith (2014) or (Smith, 2014)

Two authors: Smith and Jones (2014) or (Smith and Jones, 2014)

Three or more authors: Dupondt et al. (2014) or (Dupondt et al., 2015)

When several in-text references occur at the same point, give them in chronological order separated by semicolons.

Reference list

At the end of a document, list the references to sources that have been cited within the text, including those found in tables and figures, under the heading References.

To speed the processing of the manuscript, we strongly recommend the authors to check very carefully reference list $v s$ references in the text and tables.

References with two or more authors should be arranged first alphabetically then chronologically. The names of cited journals should be given in full.

Book: Smith A. J., 2012. Forest ecosystems. Abingdon, Routledge, 498 p.

Chapter: Smith A. J, Jones B. D., 2014. Hawaii new forest ecosystems. In: Tropical new forests, Eds McKey D. M. and Willey E. M. Abingdon, Routledge, p. 229-244.

Journal: Smith A. J, Jones B. D., 2014. Reconsidering tropical forestry. Forestry Journal, 62, 4:112-117.

If six or more authors, list the first six followed by a comma and et al.

\section{Figures}

Please provide the highest quality figure format possible. Please be sure that all imported scanned material is scanned at the appropriate resolution: $1200 \mathrm{dpi}$ for line art, $600 \mathrm{dpi}$ for grayscale and $300 \mathrm{dpi}$ for color.

Please do not embed figures in the manuscript file.

Files should be saved as one of the following formats: XLS (MS Excel file), TIFF (tagged image file format), PostScript or EPS (encapsulated PostScript).

All figures must be numbered in the order in which they appear in the manuscript (e. g. figure 1, figure 2). In multi-part figures, each part should be labelled (e. g. figure 1(a), figure 1(b)). Figure captions must be saved separately, as part of the file containing the complete text of the manuscript, and numbered correspondingly.

\section{Reproduction of copyright material}

If you wish to include any material in your manuscript in which you do not hold copyright, you must obtain written permission from the copyright owner, prior to submission. This applies to direct (verbatim or facsimile) reproduction as well as "derivative reproduction" (where you have created a new figure or table which derives substantially from a copyrighted source).

\section{Manuscript submission}

All submissions should be made using the e-mailing address of Bois et Forêts des Tropiques 0. Please note that the total size of the attached files should not exceed 7 Mo. If the whole size is higher, please use separate mailing.

Manuscripts may be submitted in any standard editable format, including Word and EndNote.

\section{Copyright and authors' rights}

To assure the integrity, dissemination, and protection against copyright infringement of published articles, you will be asked to assign us, via a Publishing Agreement, the copyright in your article. Your Article is defined as the final, definitive, and citable Version of Record, and includes the accepted manuscript in its final form, including the abstract, text, bibliography, and all accompanying tables, illustrations, data. Our Publishing Agreement with you will constitute the entire agreement and the sole understanding between you and us; no amendment, addendum, or other communication will be taken into account when interpreting your and our rights and obligations under this Agreement.

For further information, or to submit an article, please contact: E-mail: bft@cirad.fr

bft.cirad.fr 